\title{
Human papillomavirus infection is not related with prostatitis-related symptoms: results from a case- control study
}

Riccardo Bartoletti, Tommaso Cai, Enrico Meliani, Nicola Mondaini, Francesca Meacci, Patrizia Addonisio, Sara Albanese, Gabriella Nesi, Sandra Mazzoli

Department of Urology (RB, EM, NM); Department of Pathology and Oncology (GN), University of Florence, Florence; Department of Urology, Santa Chiara Regional Hospital (TC), Trento and Sexually Transmitted Diseases Center, Santa Maria Annunziata Hospital (FM, PA, SA, SM), Florence, Italy

\section{ABSTRACT}

Purpose: To investigate the relationship between human papillomavirus (HPV) infection and prostatitis-related symptoms.

Materials and Methods: All young heterosexual patients with prostatitis-related symptoms attending the same Center from January 2005 to December 2010 were eligible for this case-control study. Sexually active asymptomatic men were considered as the control group. All subjects underwent clinical examination, Meares-Stamey test and DNA-HPV test. Patients with prostatitis-related symptoms and asymptomatic men were compared in terms of HPV prevalence. Moreover, multivariable Cox proportional hazards regression analysis was performed to determine the association between HPV infection and prostatitis-related symptoms.

Results: Overall, 814 out of 2,938 patients (27.7\%) and 292 out of 1,081 controls (27.0\%) proved positive to HPV. The HPV genotype distribution was as follows: HR-HPV 478 (43.3\%), PHR-HPV 77 (6.9\%), LR-HPV 187 (16.9\%) and PNG-HPV 364 (32.9\%). The most common HPV genotypes were: 6, 11, 16, 26, 51, 53 and 81. No difference was found between the two groups in terms of HPV infection (OR 1.03; 95\% CI 0.88-1.22; $\mathrm{p}=0.66)$. We noted a statistically significant increase in HPV infection over the period 2005 to 2010 ( $p<0.001$ ) in both groups. Moreover, we found a statistically significant increase in HPV 16 frequency from 2005 to 2010 ( $p=0.002)$.

Conclusions: This study highlights that prostatitis-like symptoms are unrelated to HPV infection. Secondary, we highlight the high prevalence of asymptomatic HPV infection among young heterosexual men.

\section{ARTICLE INFO}

\section{Key words:}

Human papillomavirus 11;

Prostatitis; Diagnosis; Infection

Int Braz J Urol. 2014; 40: 247-56

Submitted for publication:

July 18, 2013

Accepted after revision:

January 15, 2014

\section{INTRODUCTION}

Human papillomavirus (HPV) infection is one of the most common sexually transmitted infections in both genders (1). HPV infection is the main cause of cervical cancer in women and is responsible for other cancers such as penile, oral-neck and anal cancer in men (2). Men are key to the transmission of HPV to women, but relatively little is known about the natural history of HPV infection in men (3). The prevalence of HPV in males ranges from $7 \%$ to $45 \%$ (4), but the majority 
of studies have been conducted on homosexuals, HIV patients or infertile men $(5,6)$. Recently, Klinglmair et al. found a high prevalence of HPV infection in a cohort of 250 young males, including children (0-10 years), indicating non-sexual transmission pathways (7). Genital infection is often asymptomatic and undiagnosed. In a recent study on 2,702 uncircumcised, HIV sero-negative males, Rositch et al. found that 51\% of them presented occult HPV infection, of whom 57\% with HPV multiple types (8). Thus, HPV prevalence data in men vary widely depending on the anatomical sites sampled, populations studied and analytical methods used for HPV detection (9). Several authors suggest that HPV tends to infect the prostatic epithelium (9), however, no correlation between symptoms from prostate pathology and HPV infection has ever been reported. On the basis of HPV DNA PCR findings, it has been suggested that the male genitourinary tract, including sperm cells, might act as a reservoir for HPV persistence and infection $(9,10)$. On the other hand, prostatitis-related symptoms are recognized as an important socio-economical problem and several sexually transmitted infections are linked to the presence of prostatitis-related symptoms $(11,12)$. We investigated HPV infection prevalence in young heterosexual males with prostatitis-related symptoms in order to find a possible relationship between HPV infection and prostatitis-related symptoms. The present case-control study aimed to determine whether HPV infection could be considered a risk factor of prostatitis-related symptoms in young heterosexual males.

\section{MATERIALS AND METHODS}

\section{Study design}

The study population consisted of two groups of young heterosexual men attending the same Sexually Transmitted Diseases Center from January 2005 to December 2010: Group A (cases), all consecutive patients with prostatitis-related symptoms, and Group B (controls), sexually active asymptomatic men whose female partners were infected with Chlamydia trachomatis. All patients and controls were screened for this study using the 4-glass Meares-Stamey test and and
DNA-HPV test. All controls underwent 4-glass Meares-Stamey test and DNA-HPV test due to the fact that they were partners of female affected by Chlamydia trachomatis infection. This is the routinely practice in our STDs Centre. Clinical and laboratory findings for the two groups were compared. This case-control study aimed to determine whether exposure to HPV infection could be associated with clinical outcomes such as the presence of prostatitis-related symptoms. This study was conducted in line with the STROBE statement (http://www.strobe-statement.org). Italian law does not require authorization from the institutional review board (IRB), nor informed consent from the patients (http://www.agenziafarmaco.gov.it/ it/content/linee-guida-studi-osservazionali). Nevertheless, our study was conducted in line with the Good Clinical Practice guidelines and with the ethical principles laid down in the latest version of the Declaration of Helsinki.

\section{Inclusion and exclusion criteria}

Group A patients were selected consecutively from a series of individuals suffering from chronic prostatitis-related symptoms for over 6 months, as defined in the latest version of the European Association of Urology (EAU) guidelines (13). In particular, the patients were selected and categorized according to Nickel's criteria for perineal or ejaculatory pain. All enrolled patients must have had a pain score equal to or greater than 4, defined as "mild prostatitis" if the pain score ranged from 4 to 7 and "moderate or severe prostatitis" if the score was 8 or greater (14). Subjects under 18 and over 45 years of age, affected by major concomitant diseases, with known anatomical abnormalities of the urinary tract or with evidence of other urological diseases, and diagnosed with genital or anal warts, participating in an HPV vaccine study, were excluded as well as patients with suspected urothelial cell carcinoma at cytological urine analysis or who had previously undergone prostate surgery. Group B patients were included in the study only if asymptomatic and falling within the age range of 18 - 45 years, and with no known major concomitant diseases. All homosexuals were excluded from both study groups due to their increased risk of exposure to 
HPV infection. Moreover, all patients have been asked about the sexual experience and the circumcision status. A detailed assessment about the sexual behavior has been carried out.

\section{Study schedule and sample collection}

We retrospectively collected anamnestic, clinical, laboratory and microbiological data for 6,743 patients from our database (Advanced PROSTATitis DataBase, Microsoft Access format). From these, we excluded 2,725 due to lack of data. Finally we considered 4,018 individuals for this study (2,938 patients and 1,081 controls) (Figure-1). 2,938 patients with prostatitis-related symptoms were assigned to Group A while 1,080 to Group B. We collected the validated Italian versions of the NIH Chronic Prostatitis Symptom Index (NIH-CPSI) (15) and the International Prostatic Symptom Score (IPSS) (16) for all patients and controls. All microbiological data were collected in accordance with indications described by Mazzoli et al. first void early morning urine (VB1), mid-stream urine (VB2), expressed prostatic se- cretion (EPS), post prostate massage urine (VB3) and total ejaculate (TE) (12). In order to evaluate the HPV infection in the urethra, a urethral swab (UR_SW) was taken from all subjects. Moreover, in our Centre we did not routinely performed the HPV specific antibodies.

\section{Laboratory procedures and microbiological con- siderations}

Microbiological cultures were performed in accordance with the methods described by Motrich et al. (17). DNA extraction and purification of all biological materials was performed using the DNeasy ${ }^{\circledR}$ Tissue Kit by QIAGEN Spa, Italy. $200 \mu \mathrm{L}$ of pellet was pre-incubated overnight with proteinase $\mathrm{k}$ and the next day extracted and purified following the manufacturer's instructions. All the biological material from the whole study population was tested for the presence of genital HPV using Alpha Watch HPV, Alphagenic-Diaco-Biotechnology, Trieste, Italy. All biological materials from our patients were analyzed by Inno-Lipa HPV Genotyping Extra (Innogenetics, Italy). Amplification of a

Figure 1 - The figure shows the Study flow-chart.

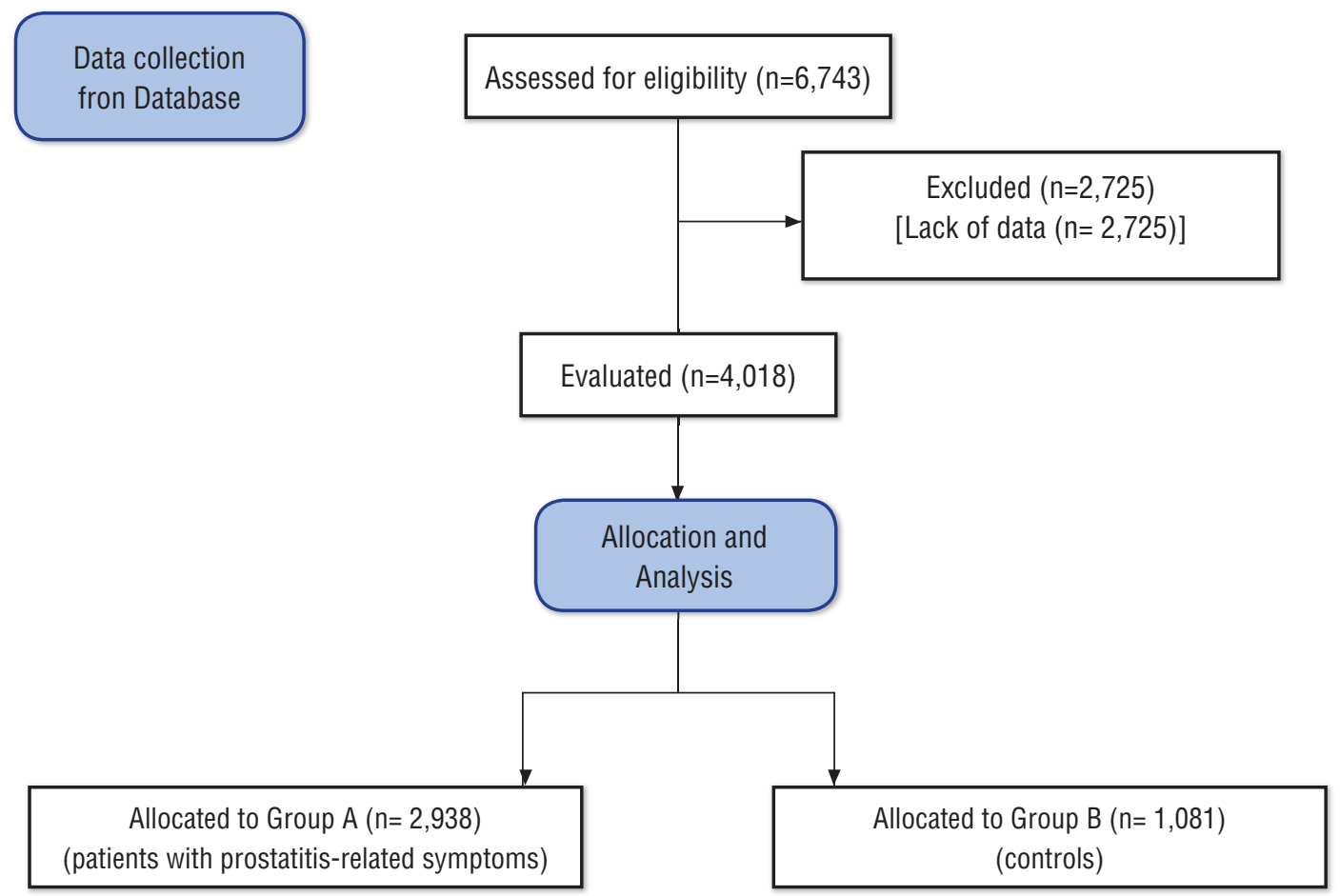


fragment of the b-globin gene served as an internal quality control for each specimen. In accordance to Munoz et al., we classified the following genotypes as high risk-HPV (HR-HPV): 16, 18, 31, 33, 35, 39, $45,51,52,56,58,59,68,73$, and 82 ; as probable high-risk (PHR-HPV): 26, 53, and 66; and as low risk (LR-HPV): 6, 11, 40, 42, 43, 44, 54, 61, 70, 72, 8118. HPV positive samples which did not hybridize with any of the type-specific probes were referred to as positive non genotype-able (PNG-HPV) (18). According to Giuliano et al., a participant was considered positive for "any HPV" if he tested HPV-positive by PCR or by genotyping (19). The category "any oncogenic type" included those who were positive for only oncogenic genotypes and those who were positive for both oncogenic and non-oncogenic types. Only single or multiple infections with non-oncogenic HPV types were classified as "any non-oncogenic type". This STDs laboratory is registered by the United Kingdom National External Quality Assessment (NEQUAS) for microbiology for molecular detection of Ct (Quality Assurance Laboratory, Health Protection Agency Centre for Infection, 61 Colindale Avenue, London NW 95HT, United Kingdom). All microbiological analyses were performed blindly. Following Nickel et al., white blood cell (WBC) counts in all biological samples were carried out, though not considered in this study (20).

\section{Statistical analysis and considerations}

Pearson's coefficient was adopted to evaluate the correlation between the different parameters in all patients, and Fisher's exact test or Chi-square test $\left(\chi^{2}\right)$ used to assess statistical significance with $\mathrm{p}<0.05$ accepted as significant. Multivariable Cox proportional hazards regression analysis was then performed to determine the association between HPV infection and prostatitis-related symptoms. The ANOVA test was applied to evaluate the difference between the two groups in terms of NIH-CPSI and IPSS questionnaire scores. The Bonferroni adjustment test was also used at the second stage of the analysis of variance. Odds ratio (OR) and 95\% confidence intervals (CI) were calculated to determine the significance of differences. Statistical significance was set at $p<0.05$. All reported $\mathrm{p}$-values are two-sided. All statistical analyses were performed by using SPSS 11.0 for Apple-Macintosh (SPSS, Inc., Chicago, Illinois).

\section{RESULTS}

Data from 4,018 subjects were collected and analysed. A total of 2,938 patients were assigned to Group A (cases, prostatitis-related symptoms) while 1,080 to Group B (controls, asymptomatic subjects).

\section{Clinical and microbiological evaluation}

Detailed information about demographic and socioeconomic variables, medical history and clinical data at enrollment are given in Table-1. All cases revealed a mean symptom time of 15.4 months (range 10-26 months) but none presented genital or anal warts on physical examination. Overall, 814 out of the 2,938 patients (27.7\%) and 292 out of the 1,081 controls $(27.0 \%)$ proved positive to HPV (Odds ratio [OR] 1.03; 95\% CI 0.88-1.22; $\mathrm{p}=0.66$ ). The HPV genotype distribution is as follows: HR-HPV 478 (43.3\%), PHR-HPV 77 (6.9\%), LR-HPV 187 (16.9\%) and PNG-HPV 364 (32.9\%). Data stratification according to age is given for both groups in Tables 2 and 3. In Group A, 28 out of 814 (3.4\%) were also positive to Chlamydia trachomatis vs 31 out of 292 (10.6) in Group B, with a statistically significant difference $(p<0.001$; Chi square 20.5; $\mathrm{df}=1)$.

HPV prevalence and genotype distribution (Group A)

Patients with prostatitis-related symptoms

HPV genotype distribution was as follows: HR-HPV 417 (51.3\%), PHR-HPV 70 (8.7\%), LR-HPV 64 (7.7\%) and PNG-HPV 263 (32.3\%). The most common HPV genotypes were: 16 (18\%), 31 (29\%) and 33 (24\%). HPV genotype distribution stratified per year is detailed in Table-2. HPV 16 and PNG-HPV frequencies were found to significantly increase over the period 2005 to 2010 ( $p=0.002$ and $p=0.003$, respectively). No statistically significant increase in frequency was found for the other HPV types. In addition, there was a decrease in HR-HPV and PHR-HPV incidence in 2009.

\section{Asymptomatic patients (Group B)}

HPV genotype distribution for asymptomatic subjects was as follows: HR-HPV 116 
Table 1 - Patient's sociodemographic anamnestic, clinical characteristics at enrolment time.

\begin{tabular}{|c|c|c|c|}
\hline \multirow[t]{3}{*}{ No. of total enrolled subjects } & \multicolumn{3}{|c|}{4,019} \\
\hline & Patients & Controls & \\
\hline & 2,938 & 1,081 & $p$ \\
\hline Median age $\left( \pm S D^{\star}\right)$ & $35.7 \pm 5.8$ & $36.0 \pm 5.9$ & 0.14 \\
\hline \multicolumn{4}{|l|}{ Educational level } \\
\hline Primary school & - & - & \\
\hline Secondary school & $2,001(68.1)$ & $716(66.2)$ & 0.25 \\
\hline Post-secondary education & 937 (31.9) & $365(33.8)$ & \\
\hline Sexually active (past month) & 2,899 (98.6) & $1,070(99.0)$ & 0.45 \\
\hline \multicolumn{4}{|l|}{ Sexual behavior } \\
\hline 1 partner & 2,376/2,899 (81.9) & $881 / 1,070$ (82.3) & 0.81 \\
\hline$>1$ partners & $523 / 2,899(18.1)$ & $189 / 1,070(17.7)$ & \\
\hline Contraceptive use & $1,903 / 2,899(65.6)$ & $693 / 1,070(64.7)$ & 0.59 \\
\hline Condom & $1,302 / 1,903(68.5)$ & 479/693 (69.2) & 0.77 \\
\hline Coitus interruptus & $601 / 1,903(31.5)$ & 214/693 (29.8) & \\
\hline \multicolumn{4}{|l|}{ Clinical data } \\
\hline \multicolumn{4}{|l|}{ Clinical presentation } \\
\hline Dysuria & $1,292(43.9)$ & - & - \\
\hline Urgency & $1,478(50.3)$ & - & - \\
\hline Dysuria + Frequency & $1,123(38.2)$ & - & - \\
\hline Burning & $981(33.3)$ & - & - \\
\hline \multicolumn{4}{|l|}{ Pain } \\
\hline Perineal & $1,341(45.6)$ & - & - \\
\hline Scrotal & $742(25.2)$ & - & - \\
\hline Suprapubic & $311(10.5)$ & - & - \\
\hline Lower Abdominal & $214(7.2)$ & - & - \\
\hline Start of CP\# history (months) & $15.4 \pm 8.2$ & - & - \\
\hline \multicolumn{4}{|c|}{ Symptoms Score at baseline (mean) (range) } \\
\hline $\mathrm{NIH}-\mathrm{CSPI}$ & $18.9(13-26)$ & $2.8(0-3)$ & $<0.001$ \\
\hline IPSS† & $17.3(1-24)$ & $2.2(0-6)$ & $<0.001$ \\
\hline
\end{tabular}

SD* = Standard Deviation; $\mathbf{C P} \#$ = Chronic prostatitis; NIH-CSPI§ = NIH- Chronic Prostatitis Symptom Index; IPSS† = International Prostatic Symptom Score. 
Table 2 - Summary results for grouped HPV type distribution by years (Patients).

\begin{tabular}{|c|c|c|c|c|c|c|c|}
\hline & \multicolumn{6}{|c|}{ Year } & \multirow[t]{2}{*}{ Total } \\
\hline & 2005 & 2006 & 2007 & 2008 & 2009 & 2010 & \\
\hline Number of patients & 584 & 446 & 523 & 547 & 427 & 411 & 2,938 \\
\hline HPV positive & $86(14.7)$ & $123(27.5)$ & $112(21.4)$ & $183(33.4)$ & $134(31.3)$ & $176(42.8)$ & $814(27.7)$ \\
\hline \multicolumn{8}{|c|}{ Sub-analysis on HPV positive patients } \\
\hline HR-HPV & $62(72.0)$ & $81(65.8)$ & $79(70.6)$ & $86(46.9)$ & $26(19.4)$ & $83(47.1)$ & $417(51.2)$ \\
\hline PHR-HPV & $5(5.8)$ & $11(8.9)$ & $5(4.4)$ & $20(10.9)$ & $5(3.8)$ & $24(13.6)$ & $70(8.6)$ \\
\hline LR-HPV & $4(4.6)$ & $7(5.7)$ & $8(7.2)$ & $12(6.6)$ & $24(17.9)$ & $9(5.2)$ & $64(7.8)$ \\
\hline PNG-HPV & $15(17.4)$ & $24(19.6)$ & $20(17.8)$ & $65(35.6)$ & $79(58.9)$ & $60(34.1)$ & $263(32.4)$ \\
\hline
\end{tabular}

HR-HPV = High-Risk HPV; PHR-HPV = Probable High-Risk HPV; LH-HPV = Low-Risk HPV; PNG-HPV = Positive Non Genotype-able HPV.

Table 3 - Summary results for grouped HPV type distribution by years (Controls).

\begin{tabular}{|c|c|c|c|c|c|c|c|}
\hline & \multicolumn{6}{|c|}{ Year } & \multirow[t]{2}{*}{ Total } \\
\hline & 2005 & 2006 & 2007 & 2008 & 2009 & 2010 & \\
\hline Number of patients & 205 & 171 & 189 & 165 & 134 & 217 & 1,081 \\
\hline HPV positive & $33(16.0)$ & $42(24.5)$ & 37 (19.5) & $51(30.9)$ & $39(29.1)$ & $90(41.4)$ & $292(27.0)$ \\
\hline \multicolumn{8}{|c|}{ Sub-analysis on HPV positive patients } \\
\hline HR-HPV & $15(45.3)$ & $18(42.8)$ & $17(45.9)$ & $19(37.2)$ & $8(20.5)$ & $39(43.3)$ & $116(39.7)$ \\
\hline PHR-HPV & $4(12.1)$ & $4(9.5)$ & $5(13.5)$ & $3(5.9)$ & $1(2.6)$ & $9(10)$ & $26(8.9)$ \\
\hline LR-HPV & $2(6.0)$ & $5(11.9)$ & $3(8.1)$ & $9(17.6)$ & $2(5.2)$ & $5(5.5)$ & $26(8.9)$ \\
\hline PNG-HPV & $12(36.6)$ & $15(35.8)$ & $12(32.5)$ & $20(39.3)$ & $28(71.7)$ & 37 (41.2) & $124(42.5)$ \\
\hline
\end{tabular}

HR-HPV = High-Risk HPV; PHR-HPV = Probable High-Risk HPV; LH-HPV = low-risk HPV; PNG-HPV = positive non genotype-able HPV.

(39.7\%), PHR-HPV 26 (8.9\%), LR-HPV 26 (8.9\%) and PNG-HPV 124 (42.5\%). The most common HPV genotypes were: 11 (17.2\%) and 31 (30.2\%). Table-3 shows HPV genotype distribution per year. There was no statistically significant increase in PCN-HPV prevalence from 2005 to 2010 ( $p=0.68)$. As in Group A, we found a decrease in HR-HPV and PHR-HPV incidence in 2009. There was a statistically significant increase in HPV prevalence from 2005 to 2010 ( $p<0.001)$ in both patients and controls (Figure-2).
On the other hand, there was no increase in HR-HPV infection prevalence from 2005 to 2010 (Figure-3).

HPV incidence and distribution per biological sample

We collected 18,351 samples from the all patients. We found 2,276 positive samples from 1,106 subjects (814 Group A and 292 Group B) (Table-4). There was no difference in HPV detection rate among VB1, TE and UR_SW were found ( $p=0.30)$, nor be- 
Figure 2 - The figure shows the prevalence of HPV-positive patients and the High-Risk-HPV positive patients in both patients and controls.

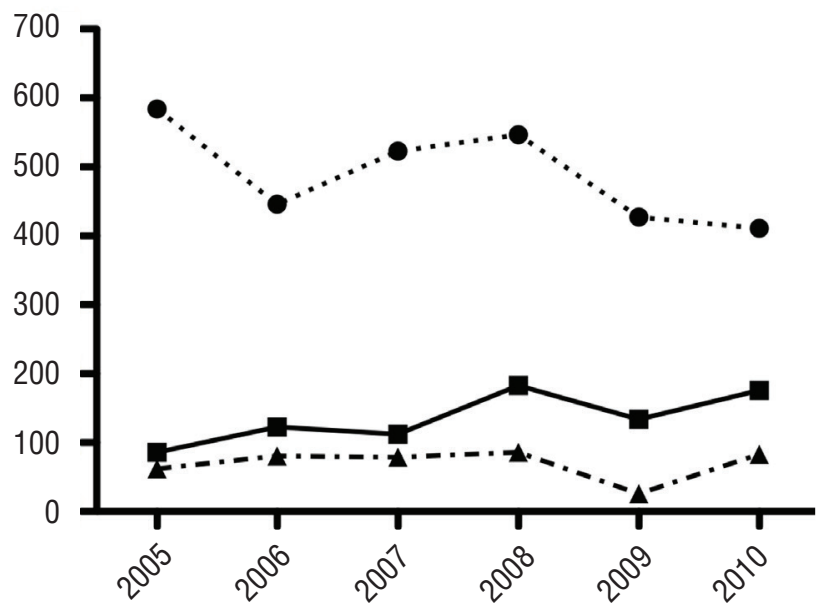

- Total Patients

- HPV positive patients

- $\mathbf{A}$ - HR-HPV positive patients

tween VB1 and UR_SW ( $\mathrm{p}=0.69)$. We also noted a statistically significant difference among VB1, VB2 and VB3 $(\mathrm{p}<0.001)$ (Figure-4). We did not include the EPS data in the analysis due to the limited number of samples collected (81 out of 814 patients for Group A, 18 out of 292 controls for Group B).
Figure 3 - The figure shows the prevalence of all HPVpositive patients stratified by risk class.

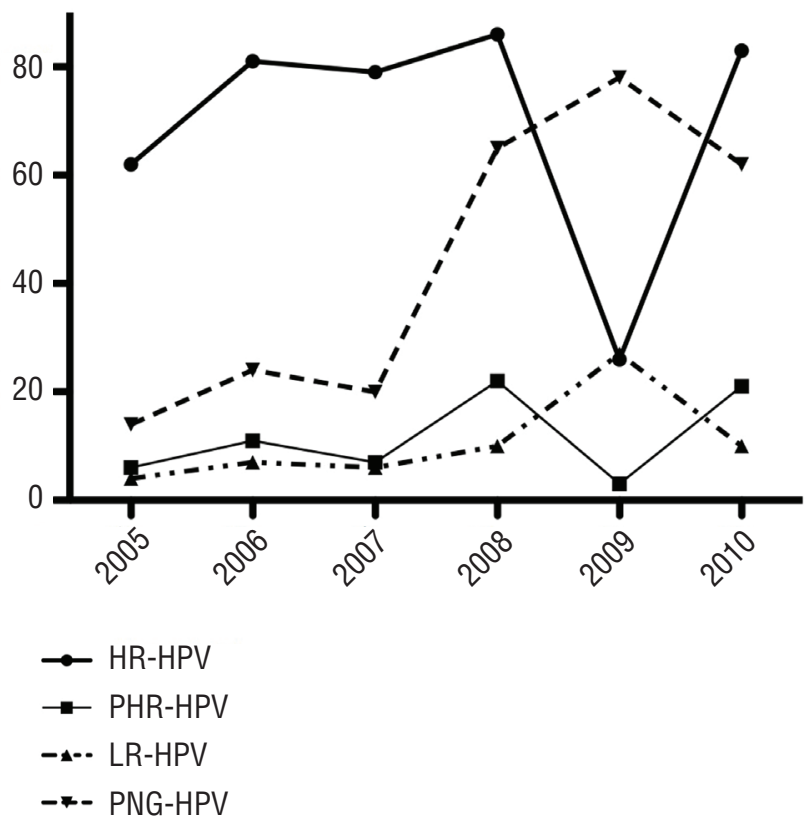

HPV and other related risk factors

No significant difference in overall HPV prevalence was found in the different age categories in either group (Table-1). Finally, we found a higher HR-HPV prevalence in Group A compared with Group B patients $(39.7 \%$ vs $51.2 \%)(p=0.008)$.

Table 4 - HPV prevalence according to collected biological samples and years.

\begin{tabular}{lccccccc}
\hline Sample & & VB1 & VB2 & VB3 & TE & UR_SW & \\
\hline & Year & & & & & & Total \\
\hline & 2005 & 77 & 2 & 8 & 69 & 81 & 237 \\
& 2006 & 119 & 5 & 18 & 112 & 118 & 372 \\
& 2007 & 101 & 9 & 14 & 108 & 105 & 337 \\
& 2008 & 161 & 12 & 27 & 115 & 169 & 484 \\
& 2009 & 123 & 6 & 11 & 99 & 129 & 368 \\
& 2010 & 163 & 5 & 19 & 121 & 170 & 478 \\
\hline Total & 744 & 39 & 97 & 624 & 772 & 2,276 \\
\hline
\end{tabular}

VB1 = First void early morning urine; VB2 = Mid-stream urine; VB3 = Expressed prostatic secretion; TE = Total ejaculate; EPS = Expressed prostatic secretion; UR_SW = Urethral swab. 
Figure 4 - The figure shows the prevalence of HPV-positive biological samples according to each analyzed sample.

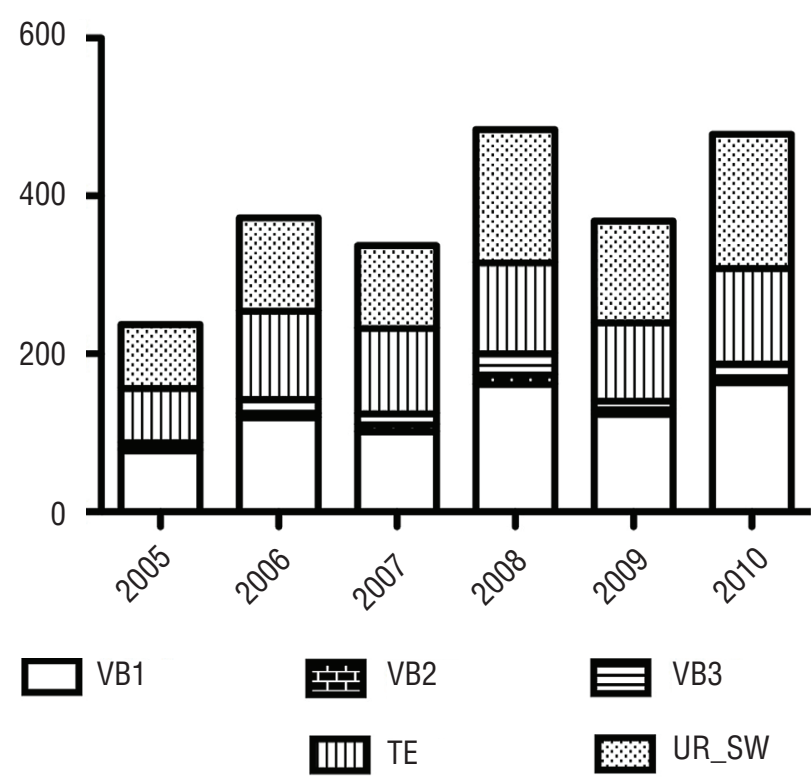

There was no difference in terms of sexual behavior or use of contraceptives (Table-1).

\section{DISCUSSION}

HPV transmission normally occurs through sexual intercourse with commonly recognized infection distribution. In the absence of any obvious clinical manifestations, such as genital warts, a male is often considered as a healthy disease-carrier (21). Genital infection is often asymptomatic in both genders, although genital warts can generate local symptoms such as burning, itching and occasional bleeding (22). In the present study, no significant difference in prevalence of HPV infection-related symptoms was found between the two groups, although approximately 33\% of Group A patients complained of burning during micturition. This symptom should be considered part of chronic pelvic pain syndrome, since all these patients had VB2 samples negative for bacteria at the 4-glass Meares-Stamey test. The cohort of young heterosexual males with prostatitis-related symptoms was selected on the basis of the recent literature on HPV reporting a high prevalence of infection (over 70\% of cases) in men between 17 and 45 years of age. Similarly, the control group was selected from asymptomatic and sexually active males, because their female sexual partners were infected with Chlamydia trachomatis, thus more exposed to the risk of developing sexually transmitted infections. Homosexuals were excluded from the study due to their increased risk of developing HPV infection (23). Several authors have demonstrated the presence of HPV in the semen, although none of them has suggested any relationship with male infertility. Perino et al. showed that the abortion rate in male-infected couples is significantly higher (66.7\%) than in normal couples (15\%) (24). Indeed, viral bodies adhere to spermatozoa, subsequently reducing their motility (25). HPV could then be transmitted from men to women during sexual intercourse through sexual "contact" as well as through biological fluids. Moreover infection of the seminal tract (prostate, seminal vesicle, vas deferens) could also be hypothesized on the basis of HPV presence in the semen of asymptomatic patients. Our results partially contradict this hypothesis. The HPV prevalence was higher in VB1 and urethral swabs than in VB2 and VB3 samples. Several authors report HPV prevalence in the urethra ranging from 20 to $48 \%$, although these data could be influenced by either the limited number of subjects evaluated, the wide age range of selected subjects or the difference in their sexual habits, activity and attitudes. On the other hand, for the same reasons, the prevalence of HPV infection in the semen has been investigated by various authors with conflicting results. Foresta et al. found $40.9 \%$ prevalence in subjects with infected sexual partners and $2.2 \%$ in fertile controls, while Nielsen et al. found 5.3\% prevalence in 463 heterosexual men $(26,27)$. Our study confirmed the high prevalence of HPV infection (27.7\%) in a large cohort of young heterosexual men and an increasing positive trend of HPV infection over a 5 year period. No significant difference in prevalence was found between the group with prostatitis-related symptoms and the asymptomatic control group, although Group A patients exhibited an increased prevalence in HR-HPV genotypes. Despite the lack of differences in sexual behavior or contraceptive use between the two groups, the control group would have been expected to show a higher rate of HR-HPV infection due to their increased risk of developing Chlamydia 
trachomatis co-infection, commonly considered a sexually transmitted infection. HPV infection prevalence in the control group was independent from Chlamydia trachomatis co-morbidity (28). These results confirm that an increased risk of developing HPV infection in young heterosexual men is not related to the number of sexual partners but to the social diffusion of the disease in both genders. This is also supported by the increasing trend of HPV infection over the five-year period. The present study shows few limitations that should be taken into account. Firstly, the retrospective nature of this study. Secondly, the highly selected patient population due to the fact that we have enrolled patients attending a specific STDs Centre. Finally, the fact that we have no data about the impact of female vaccination on HPV male prevalence.

\section{CONCLUSIONS}

In conclusion, we found no correlation between the presence of prostatitis-related symptoms and HPV infection, highlighting an asymptomatic and increasing prevalence of HPV in young heterosexual males.

\section{ACKNOWLEDGEMENTS}

We are grateful to all members of the Santa Maria Annunziata STD Centre for their assistance and to Professor John Denton for manuscript language revision.

\section{CONFLICT OF INTEREST}

None declared.

\section{REFERENCES}

1. Giuliano AR, Lee JH, Fulp W, Villa LL, Lazcano E, Papenfuss MR, et al.: Incidence and clearance of genital human papillomavirus infection in men (HIM): a cohort study. Lancet. 2011; 377: 93240. Erratum in: Lancet. 2011; 377: 2006.

2. Kjaer SK, Munk C, Winther JF, Jørgensen HO, Meijer CJ, van den Brule AJ: Acquisition and persistence of human papillomavirus infection in younger men: a prospective followup study among Danish soldiers. Cancer Epidemiol Biomarkers Prev. 2005; 14: 1528-33.
3. Benevolo M, Mottolese M, Marandino F, Carosi M, Diodoro MG, et al.: HPV prevalence among healthy Italian male sexual partners of women with cervical HPV infection. J Med Virol. 2008; 80: 1275-81.

4. Repp KK, Nielson CM, Fu R, Schafer S, Lazcano-Ponce E, Salmerón J, et al.: Male human papillomavirus prevalence and association with condom use in Brazil, Mexico, and the United States. J Infect Dis. 2012; 205: 1287-93.

5. Parisi SG, Cruciani M, Scaggiante R, Boldrin C, Andreis $\mathrm{S}$, Dal Bello $\mathrm{F}$, et al.: Anal and oral human papillomavirus (HPV) infection in HIV-infected subjects in northern Italy: a longitudinal cohort study among men who have sex with men. BMC Infect Dis. 2011; 11: 150.

6. Foresta C, Garolla A, Zuccarello D, Pizzol D, Moretti A, Barzon L, et al.: Human papillomavirus found in sperm head of young adult males affects the progressive motility. Fertil Steril. 2010; 93: 802-6.

7. Klinglmair G, Pichler R, Zelger B, Dogan HS, Becker T, Esterbauer $\mathrm{J}$, et al.: Prevalence of the human papillomavirus (HPV) expression of the inner prepuce in asymptomatic boys and men.World J Urol. 2013; 31: 1389-94.

8. Rositch AF, Poole C, Hudgens MG, Agot K, Nyagaya E, Moses S, et al.: Multiple human papillomavirus infections and type competition in men. J Infect Dis. 2012; 205: 72-81.

9. Tachezy R, Hrbacek J, Heracek J, Salakova M, Smahelova J, Ludvikova V, et al.: HPV persistence and its oncogenic role in prostate tumors. J Med Virol. 2012; 84: 1636-45.

10. Veldhuijzen NJ, Snijders PJ, Reiss P, Meijer CJ, van de Wijgert $\mathrm{JH}$ : Factors affecting transmission of mucosal human papillomavirus. Lancet Infect Dis. 2010; 10: 862-74.

11. Bartoletti R, Cai T, Mondaini N, Dinelli N, Pinzi N, Pavone $\mathrm{C}$, et al.: Prevalence, incidence estimation, risk factors and characterization of chronic prostatitis/chronic pelvic pain syndrome in urological hospital outpatients in Italy: results of a multicenter case-control observational study. J Urol. 2007; 178: 2411-5; discussion 2415.

12. Mazzoli S, Cai T, Rupealta V, Gavazzi A, Castricchi Pagliai $\mathrm{R}$, Mondaini $\mathrm{N}$, et al.: Interleukin 8 and anti-chlamydia trachomatis mucosal $\lg A$ as urogenital immunologic markers in patients with $\mathrm{C}$. trachomatis prostatic infection. Eur Urol. 2007; 51: 1385-93.

13. Naber KG, Bergman B, Bishop MC, Bjerklund-Johansen TE, Botto $H$, Lobel $B$, et al.: EAU guidelines for the management of urinary and male genital tract infections. Urinary Tract Infection (UTI) Working Group of the Health Care Office (HCO) of the European Association of Urology (EAU). Eur Urol. 2001; 40: 576-88.

14. Nickel JC, Downey J, Hunter D, Clark J: Prevalence of prostatitis-like symptoms in a population based study using the National Institutes of Health chronic prostatitis symptom index. J Urol. 2001; 165: 842-5. 
15. Giubilei G, Mondaini N, Crisci A, Raugei A, Lombardi $G$, Travaglini $F$, et al.: The Italian version of the National Institutes of Health Chronic Prostatitis Symptom Index. Eur Urol. 2005; 47: 805-11.

16. Badía X, García-Losa M, Dal-Ré R: Ten-language translation and harmonization of the International Prostate Symptom Score: developing a methodology for multinational clinical trials. Eur Urol. 1997; 31: 129-40.

17. Motrich RD, Cuffini C, Oberti JP, Maccioni M, Rivero VE: Chlamydia trachomatis occurrence and its impact on sperm quality in chronic prostatitis patients. J Infect. 2006; 53: 175-83.

18. Muñoz N, Bosch FX, de Sanjosé S, Herrero R, Castellsagué $X$, Shah KV, et al.: Epidemiologic classification of human papillomavirus types associated with cervical cancer. N Engl J Med. 2003; 348: 518-27.

19. Giuliano AR, van der Loeff MF, Nyitray AG: Circumcised HIVinfected men and HPV transmission. Lancet Infect Dis. 2011; 11: $581-2$.

20. Nickel JC, Alexander RB, Schaeffer AJ, Landis JR, Knauss JS, Propert KJ; et al.: Leukocytes and bacteria in men with chronic prostatitis/chronic pelvic pain syndrome compared to asymptomatic controls. J Urol. 2003; 170: 818-22.

21. Bartoletti R, Zizzo G, Cai T, Mirone V: Genital condylomata are not the human papilloma virus male infection burden. Eur Urol. 2011; 60: 268-9.

22. Shi JF, Kang DJ, Qi SZ, Wu HY, Liu YC, Sun LJ, et al.: Impact of genital warts on health related quality of life in men and women in mainland China: a multicenter hospital-based cross-sectional study. BMC Public Health. 2012; 12: 153.

23. Machalek DA, Grulich AE, Jin F, Templeton DJ, Poynten IM: The epidemiology and natural history of anal human papillomavirus infection in men who have sex with men. Sex Health. 2012; 9: 527-37.
24. Perino A, Giovannelli L, Schillaci R, Ruvolo G, Fiorentino FP, Alimondi $P$, et al:: Human papillomavirus infection in couples undergoing in vitro fertilization procedures: impact on reproductive outcomes. Fertil Steril. 2011; 95: 1845-8.

25. Foresta C, Patassini C, Bertoldo A, Menegazzo M, Francavilla F, Barzon L, et al.: Mechanism of human papillomavirus binding to human spermatozoa and fertilizing ability of infected spermatozoa. PLoS One. 2011; 6: e15036.

26. Foresta C, Pizzol D, Moretti A, Barzon L, Palù G, Garolla A: Clinical and prognostic significance of human papillomavirus DNA in the sperm or exfoliated cells of infertile patients andsubjects with risk factors. Fertil Steril. 2010; 94: 1723-7.

27. Nielson CM, Harris RB, Flores R, Abrahamsen M, Papenfuss MR, Dunne EF, et al.: Multiple-type human papillomavirus infection in male anogenital sites: prevalence and associated factors. Cancer Epidemiol Biomarkers Prev. 2009; 18: 1077-83.

28. Cai T, Wagenlehner FM, Mondaini N, D’Elia C, Meacci F, Migno S, et al.: Effect of human papillomavirus and Chlamydia trachomatis co-infection on sperm quality in young heterosexual men with chronic prostatitis-related symptoms. BJU Int. 2013. In press.
Correspondence address: Tommaso Cai, MD Department of Urology Santa Chiara Regional Hospital Largo Medaglie d'Oro, 9 Trento, 38123, Italy E-mail: ktommy@libero.it 